\title{
Monozygotic twins discordant for congenital adrenal hyperplasia due to mosaicism
}

\author{
Michelle L Kluge', Evan Graber², Kathryn Foley³, Lynnette V Hansen'1, Heidi L Sellers', Dragana Milosevic', \\ Kendall W Cradic ${ }^{1}$ and Stefan K Grebe ${ }^{1}$ \\ ${ }^{1}$ Department of Laboratory Medicine and Pathology, Mayo Clinic, Rochester, Minnesota, USA, ${ }^{2}$ Division of Pediatric \\ Endocrinology, and ${ }^{3}$ Department of Pediatrics, Nemours/AI DuPont Hospital for Children, Wilmington, \\ Delaware, USA \\ Correspondence \\ should be addressed \\ to M L Kluge \\ Email \\ kluge.michelle@mayo.edu
}

\begin{abstract}
Introduction: Genotype-phenotype discordance occurs occasionally in congenital adrenal hyperplasia (CAH). Its causes are largely unknown. We describe a case of monochorionic, diamniotic twins with discordant clinical presentations of $\mathrm{CAH}$, and show evidence for this being due to mosaicism resulting from a postzygotic full gene deletion of CYP21A2 prior to twinning.

Case description: A 7-day-old 36-week gestation female infant (Twin A) presented to the emergency department with elevated 17-hydroxyprogesterone (17-OHP). Her identical twin (Twin B) had normal 17-OHP on newborn screening. Both twins showed signs of virilization, more pronounced in Twin B. Molecular genetic testing of both twins and their parents showed a WT paternally-inherited CYP21A2 and a maternally-inherited copy containing the c.293$13 \mathrm{C}>\mathrm{G}$ mutation. Both twins were also found to have a 5'-CYP21A1P/CYP21A2-3' hybrid (product of the common 30-kb deletion), derived from the deletion of the paternally-inherited CYP21A2. Neither mother nor father carried the deletion.

Conclusions: The genetic findings are consistent with mosaicism for two CYP21A2 cell lines in both twins. The first cell line is expected, based on parental results, while the second line is due to a postzygotic full gene deletion of the paternally-inherited WT CYP21A2. The resultant genotype, compound heterozygosity for c.293-13C>G and a CYP21A2 full gene deletion, is consistent with a salt-wasting CAH phenotype. Differential distribution of the second cell line between the twins is most likely the cause for their discrepant phenotypes. We believe this is the first report of somatic CYP21A2 mosaicism, and represents a novel cause for discrepant CAH phenotypes in monozygotic twins.
\end{abstract}

\section{Introduction}

Congenital adrenal hyperplasia (CAH) due to 21-hydroxylase deficiency is an autosomal recessive condition of variable severity, which results from pathogenic CYP21A2 mutations. The underlying genetic defects can be complex, and unequal crossover between CYP21A2 and its highly homologous pseudogene CYP21A1P may result in deletion (or duplication) of the CYP21A2 gene or a gene conversion event, where a section of CYP21A1P is copied onto CYP21A2 (1). Indeed, gene deletions, gene conversion events (hybrid alleles) and pseudogene-derived mutations (mutations present on the pseudogene that are transposed onto CYP21A2 by a gene conversion) account for approximately 90-95\% of CYP21A2 mutant alleles in Western Europeans $(1,2)$. In addition, approximately $1 \%$ of CYP $21 A 2$ mutations are de novo and, therefore, not found in either parent (1). Nonetheless, when appropriate genetic testing methods and expertise are employed, there is a strong genotype-phenotype correlation $(1,2,3,4,5,6,7)$. When discordance is observed, it frequently involves the 
common pathogenic mutations p.P31L, c.293-13C>G and p.I173N (legacy nomenclature: p.P30L; g.655A/C>G, IVS2-13A/C>G or I2G; and p.I172N) (2, 3, 4, 7). Genotypephenotype discordance has also occasionally been noted in siblings with identical genotypes, a phenomenon that is poorly understood $(5,6)$.

In this report, we describe a case of monochorionic, diamniotic twins with discordant clinical presentations of 21-hydroxylase deficient CAH. We hypothesize that their discrepant phenotypes are most likely due to mosaicism resulting from a postzygotic full gene deletion of their paternally-inherited copy of CYP21A2 $(8,9)$. Although Marino et al. reports of a suspected case of germline mosaicism in the unaffected father of two affected siblings sharing the same de novo mutation, the authors are unaware of any previous reports of mosaicism as a cause of discrepant CAH presentation in monozygotic twins (6). Nor are the authors aware of a previous report of somatic mosaicism for a mutation in CYP21A2 giving rise to 21-hydroxylase deficient CAH. Postzygotic mutations resulting in mosaicism and discordant phenotypes in monozygotic twins have been reported in other hereditary conditions $(8,9)$.

\section{Case}

\section{Clinical and biochemical presentation}

A 7-day-old ex-36 week female infant (Twin A) presented to the emergency department with elevated 17-hydroxyprogesterone (17-OHP) by newborn screening (NBS; $86.4 \mathrm{ng} / \mathrm{mL}$, normal: <30) drawn at $34 \mathrm{~h}$ of life. Her identical twin (Twin B) had normal 17-OHP by NBS (0.8 $\mathrm{ng} / \mathrm{mL})$.

Follow-up serum studies on the sixth postnatal day revealed 17-OHP concentrations of $7840 \mathrm{ng} / \mathrm{dL}$ and $<40 \mathrm{ng} / \mathrm{dL}$, respectively (normal: <630 ng/dL). Serum sodium was low in Twin A and normal in Twin B (131 $\mathrm{mEq} / \mathrm{L}$ versus $141 \mathrm{mEq} / \mathrm{L}$, normal: 134-144), while serum potassium was substantially elevated in Twin A (8.7 $\mathrm{mEq} / \mathrm{L}$, normal 3.5-5.2), but almost normal in Twin B (5.6 mEq/L).

Androstenedione, renin activity, and testosterone for Twin A are shown in Table 1. Androstenedione, renin activity and testosterone were not tested for Twin B. An ACTH (adrenocorticotropic hormone) stimulation test was not performed for either twin. All hormonal measurements were performed by liquid chromatographytandem mass spectrometry.

Twin A was started on hydrocortisone, fludrocortisone, and sodium chloride supplementation with gradual resolution of hyperkalemia, hyponatremia, and a decline in 17-OHP serum concentrations.

Initial physical examination of both twins at age 10 days revealed mild to moderate clitoromegaly. Twin A was noted to have a phallus length of $1.5 \mathrm{~cm}$, and Twin B was noted to have a phallus length of $1.0 \mathrm{~cm}$, width of $4.0 \mathrm{~mm}$, and an anogenital distance of $2.5 \mathrm{~cm}$ (normal: $9.1 \pm 2.8 \mathrm{~mm}$ in full term infant). Hyperpigmentation of the labia majora was present in both twins, along with posterior labial fusion, which was noted to be slight in Twin A and more pronounced in Twin B. Both twins also appeared to have a urogenital sinus. No gonads were palpable in the labia majora or inguinal canals. Subsequent evaluation showed that Twin A had normal vaginal and urethral opening, while Twin B was confirmed to have a urogenital sinus.

Twin A is being treated with hydrocortisone and fludrocortisone, and has required gradual increases in hydrocortisone with age. Her growth and development have been normal, and no surgical intervention has been required. Twin B has not required any hormonal treatment; urethroplasty and vaginoplasty were performed for distal urogenital sinus at 17 months of age. No other follow-up has occurred for Twin B.

Table 1 17-OHP, androstenedione, renin activity, and testosterone measurements for Twin A over a period of 35 months.

\begin{tabular}{lc}
\hline Age at collection & $\mathbf{1 7 - O H P ~ ( n g / d L )}$ \\
\hline 6 days & $\mathbf{7 8 4 0}$ \\
17 months & $\mathbf{2 6 3 0}$ \\
20 months & $\mathbf{1 1 0 0}$ \\
23 months & $\mathbf{4 2 8 0}$ \\
27 months & 338 \\
32 months & $\mathbf{2 4 4 0}$ \\
35 months & $\mathbf{1 2 7 0}$ \\
\hline
\end{tabular}

\begin{tabular}{c}
\hline Androstenedione $(\mathrm{ng} / \mathrm{dL})$ \\
\hline \\
58 \\
$\mathbf{8 2}$ \\
$\mathbf{7 1}$ \\
$<16$ \\
32 \\
23 \\
\hline
\end{tabular}

\begin{tabular}{c}
\hline Renin Activity $(\mathrm{ng} / \mathrm{mL} / \mathrm{h})$ \\
\hline $23 *$ \\
$55^{*}$ \\
$61 *$ \\
$33^{* *}$ \\
$16 * *$ \\
$12 * *$
\end{tabular}

Testosterone $(\mathrm{ng} / \mathrm{dL})$

Bold values indicate elevated results. Normal ranges: 17-OHP: 0-28 days: $<630 \mathrm{ng} / \mathrm{dL}$, prepubertal females: $<100 \mathrm{ng} / \mathrm{dL}$; Androstenedione: $<69$ ng/dL; Renin activity: *0-2 years: 4.6 ng/mL/h (mean), range: $1.4-7.8$ ng/mL/h; **3-5 years: $2.5 \mathrm{ng} / \mathrm{mL} / \mathrm{h}$ (mean), range: $1.5-3.5 \mathrm{ng} / \mathrm{mL} / \mathrm{h}$; Testosterone: <2.5-10 $\mathrm{ng} / \mathrm{dL}$. 


\section{Genetic testing}

Zygosity testing [Mayo Clinic Laboratories (MCL), Rochester, MN] revealed a $>99.999 \%$ likelihood of monozygosity and ruled out non-paternity. Genetic testing by mini-sequencing for CYP21A2 variants (Esoterix Laboratory, Calabasas Hills, CA) on blood-derived DNA from both twins failed to clarify their clinical phenotypes, finding the same heterozygous pseudogene-derived saltwasting c.293-13C $>\mathrm{G}$ variant in both twins.

Further CYP21A2 genetic testing was performed (MCL). A multi-method approach involving PCR amplification, bi-directional sequencing analysis, and multiplex ligationdependent probe amplification (MLPA) was used to detect sequence variants and copy number [GenBank accession number NM_000500.7; build GRCh37 (hg19)] (Fig. 1 and Supplementary Table 1, see section on supplementary materials given at the end of this article).

\section{Genetic testing results}

Utilizing blood-derived DNA, PCR indicated the presence of CYP21A2, CY21A1P and a $5 '-C Y P 21 A 1 P / C$ $Y P 21 A 2-3^{\prime}$ hybrid in both twins. The $5^{\prime}-C Y P 21 A 1 P / C$ YP21A2-3' hybrid is a byproduct of the common $30-\mathrm{kb}$ CYP21A2 deletion. Copy number determination by MLPA was ambiguous, yielding 1-2 copies of CYP21A2 and 2-3 copies of CYP21A1P in both twins. This unusual result was confirmed by droplet digital PCR (ddPCR), which yielded 1.5 copies of the CYP21A2 promoter and

A

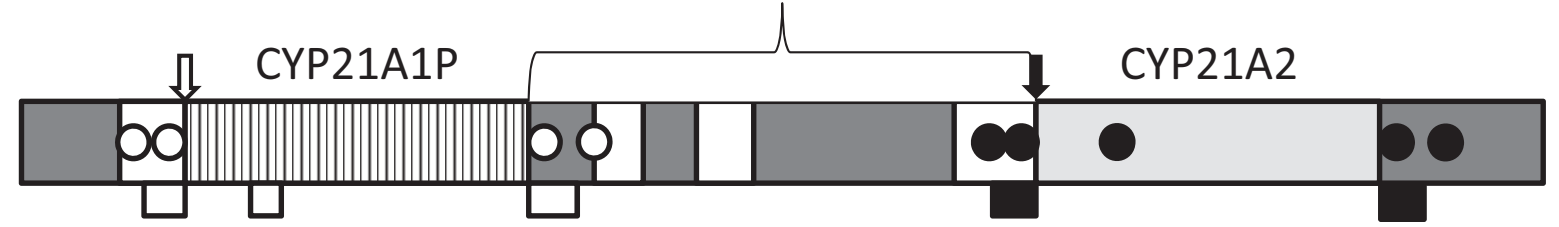

O CYP21A1P Long Range PCR Amplification Primers

CYP21A2 Long Range PCR Amplification Primers

$\square \quad$ CYP21A1P MLPA Probes

CYP21A2 MLPA Probes

I CYP21A1P ddPCR probe

$\downarrow$ CYP21A2 ddPCR probe

B

Possible long-range PCR- and corresponding MLPA results:

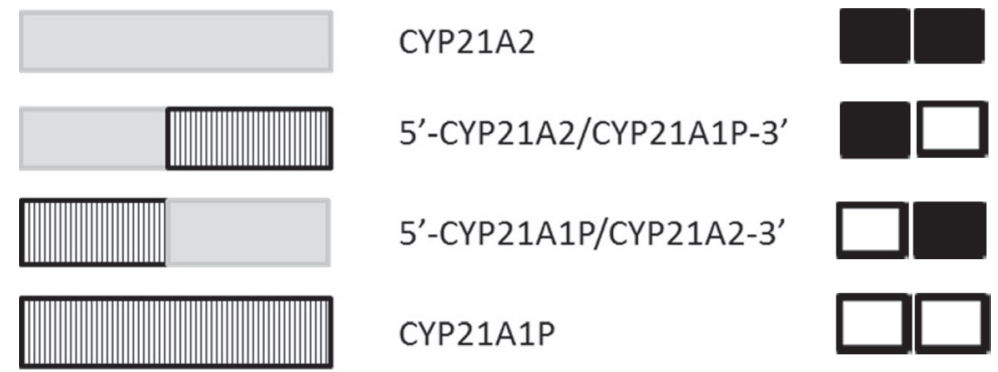

Figure 1

Schematic depiction of CYP21 locus and of the assay methodology used for diagram of the locus containing CYP21A1P and CYP21A2. (A) Relative location of CYP21A1P and CYP21A2 long range PCR amplification primers, MLPA probes, and ddPCR probes. For detailed sequences, see Supplementary Table 1. (B) Possible results based on long range PCR and MLPA probes. 
A
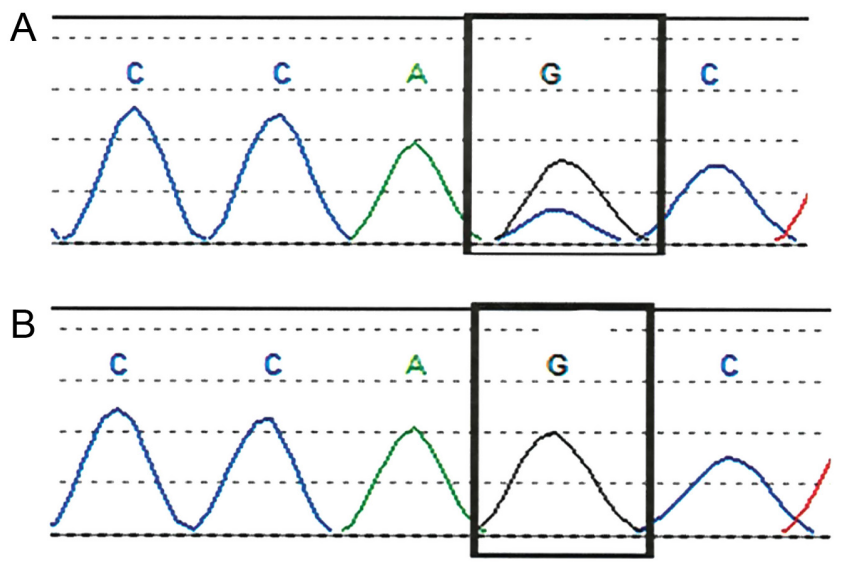

Figure 2

Sequencing of the CYP21A2 gene and the 5'-CYP21A1P/CYP 21A2-3' hybrid of Twin A. (A) Position c.293-13 in CYP21A2 (black box); sequencing demonstrates that Twin $A$ is heterozygous for both the wildtype cytosine $(C)$ in blue and the mutant guanine $(G)$ in black. Note the peak intensity for the WT $C$ is reduced compared to the peak intensity for the mutant G. (B) Position c.293-13 in the 5'-CYP21A1P/CYP21A2-3' hybrid (black box); the mutant guanine is the only nucleotide present at the c.293-13 position. This is expected since the c.293-13C>G mutation is pseudogene-derived.

2.5 copies of the CYP21A1P promoter when compared to a known normal sample ( 2 copies of CYP21A2 and 2 copies of CYP21A1P). Sanger sequencing utilizing primers specific for the CYP21A2 gene confirmed, in both twins, the presence of the heterozygous c.293-13C $>$ G mutation previously detected. However, the peak intensity of c.29313C > G was unbalanced, with the WT peak (c.293-13C) being reproducibly smaller than the mutant peak (c.29313G) (Fig. 2A). This phenomenon of unbalanced peak intensity was also noted in four common benign variants (c. $-4 \mathrm{C}>$ T, c. $293-67 \mathrm{C}>\mathrm{T}$, c. ${ }^{*} 368 \mathrm{~T}>\mathrm{C}$, c. ${ }^{*} 373 \mathrm{G}>\mathrm{A}$ ) detected in the twins. Sanger sequencing of the $5^{\prime}-C Y P 21 A 1 P / C Y P$ 21A2-3' hybrid confirmed its presence in both twins and revealed pseudogene material [including the expected c.293-13G (Fig. 2B)] up through at least the end of exon 8.

PCR and MLPA utilizing blood-derived DNA from both parents revealed each parent has 2 copies of CYP21A2 and CYP21A1P, therefore neither parent carries the 5'-CYP21A1P/CYP21A2-3' hybrid detected in the twins. Sanger sequencing of the CYP21A2 gene of the mother detected heterozygosity for the c.293-13C $>\mathrm{G}$ mutation. However, the father has two copies of WT CYP21A2. The CYP21A2 gene is known to be highly polymorphic, and the father was found to carry multiple heterozygous benign

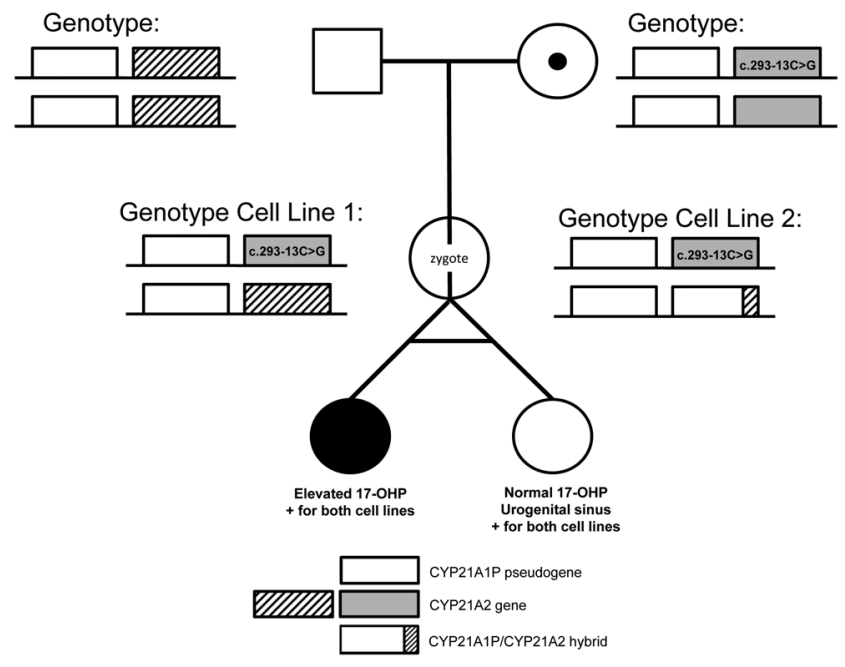

Figure 3

Pedigree chart: Circles represent females, and squares represent males. Diagnosis of congenital adrenal hyperplasia is indicated by filled-in symbols. Carrier status is denoted by a dot. The zygote prior to twinning is represented by a circle with 'zygote' printed inside. The bar between Twin A (left) and Twin B (right) reflects monozygosity. The genotype of an individual is denoted next to their symbol. Maternal and paternal CYP21A2 alleles are differentiated by gray shading and black diagonal lines respectively. The genotypes for cell lines 1 and 2 in Twin A and Twin B were determined based on the combined results of MLPA and ddPCR.

variants; these benign variants allowed for comparison with the WT copy of CYP21A2 found in the twins. One of the paternal copies of the gene (carrying five benign variants) was found to match exactly with the WT copy of CYP21A2 detected in both twins, suggesting that it was paternally inherited.

Thefour heterozygous benign variants with unbalanced peaks in the twins were investigated in the parents. It was noted that, similar to the twins' c.293-13C > G variant, the maternally-inherited nucleotide signals were reproducibly higher than the peaks corresponding to the paternallyinherited nucleotides (data not shown). Based on these results, the twins inherited one copy of CYP21A2 carrying the c.293-13C > G salt-wasting mutation from their mother and one WT copy of CYP21A2 from their father (Fig. 3). The 5'-CYP21A1P/CYP21A2-3' hybrid does not appear to have been inherited from either parent.

The $5^{\prime}-C Y P 21 A 1 P / C Y P 21 A 2-3^{\prime}$ hybrid is the typical by-product of the common $30-\mathrm{kb}$ deletion that results from unequal crossover between CYP21A2 and CYP21A1P (Fig. 4). Therefore, the 3'-UTR of the hybrid is expected 


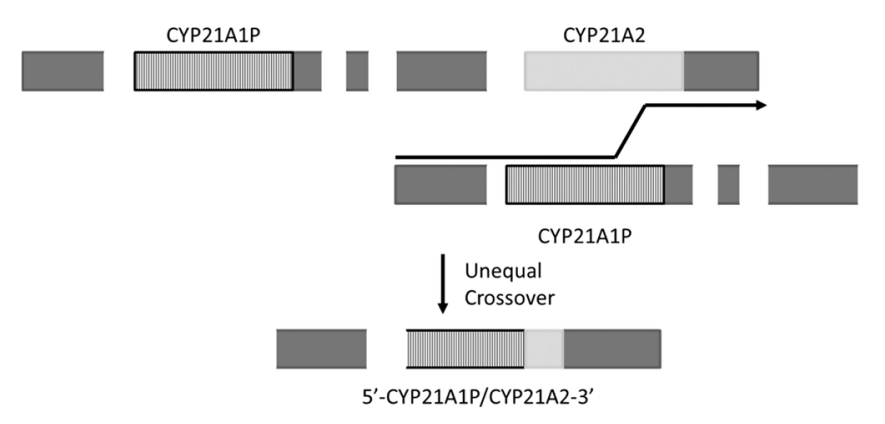

to match the 3 '-UTR of the copy of CYP21A2 that was deleted. To investigate this the 3 '-UTR of CYP21A2 was sequenced in the twins and both parents and compared to the 3'-UTR of the twins' 5'-CYP21A1P/CYP21A2-3' hybrid. Sanger sequencing of CYP21A2 revealed two heterozygous benign sequence variants in the mother: c. ${ }^{*} 368 \mathrm{~T}>\mathrm{C}$ and c. ${ }^{*} 373 \mathrm{G}>\mathrm{A}$, while the father was WT at these positions (c. ${ }^{*} 368 \mathrm{~T}$ and c.*373G) (data not shown). Both twins were heterozygous for both variants, supporting that the c. ${ }^{*} 368 \mathrm{~T}>\mathrm{C}$ and c. ${ }^{*} 373 \mathrm{G}>\mathrm{A}$ variants are in cis with the c.293-13C $>$ G variant on the maternal allele. The 3'-UTR of the $5^{\prime}-C Y P 21 A 1 P / C Y P 21 A 2-3^{\prime}$ hybrid revealed that both positions were WT (c.*368T and c.*373G), supporting that the paternally-inherited copy of CYP21A2 was deleted.

The combination of these findings suggest that the apparently de novo 5'-CYP21A1P/CYP21A2-3' hybrid resulted from a postzygotic gene conversion that occurred prior to twinning. This gene conversion event resulted in the replacement of the $5^{\prime}$ end (up through at least exon 8) of the paternally-inherited WT copy of CYP21A2 with pseudogene material. Therefore, both twins are mosaic for two cell lines. Cell line 1 is the expected cell line based on the parental results: one maternally-inherited copy of CYP21A2 that carries the c.293-13C>G mutation and one paternally-inherited WT copy of CYP21A2. Cell line 2 resulted from the gene conversion event: one maternallyinherited copy of the CYP21A2 that carries the c.293$13 \mathrm{C}>\mathrm{G}$ mutation and one de novo inactive $5^{\prime}-\mathrm{CYP} 21 \mathrm{~A} 1 \mathrm{P} / \mathrm{C}$ YP21A2-3' hybrid where the $3^{\prime}$ end of the hybrid matches the paternally-inherited copy of CYP21A2.

Germline mosaicism for the 5'-CYP21A1P/CYP21A2-3' hybrid cannot be fully ruled out in the father of the twins; however, if the father was germline mosaic, the twins would have inherited the hybrid from their father and the

\begin{abstract}
Figure 4
Unequal crossover resulting in CYP21A2 deletion. Unequal crossover and gene conversion events may occur due to the high homology of not only the CYP21A2 and CYP21A1P genes, but also other genes in this locus (RP1-C4A-CYP21A1P-TNXA and $R P 2-C 4 B-C Y P 21 A 2-T N X B)$. Unequal crossover between CYP21A1P and CYP21A2 may result in the deletion of a $30-\mathrm{kb}$ region and generation of a $5^{\prime}-C Y P 21 A 1 P / C Y$ P21A2-3' hybrid. This image is a generic example of this event, multiple breakpoints generating these hybrids or chimeras have been detected.
\end{abstract}

CYP21A2 allele carrying c.293-13C > G from their mother. A postzygotic somatic rescue event would have had to result, whereby the CYP21A2 material was copied from the available maternal allele. At that point, to match the genotype detected in the WT copy of CYP21A2 detected in the twins, five individual mutational events would have had to occur to convert the CYP21A2 gene back to the genotype detected in the twins' father. Therefore, the authors consider a postzygotic gene conversion resulting in mosaicism in the twins to be by far the most likely explanation; it would only require one event (a gene conversion).

\section{Discussion}

The results of the combined methodologies suggest a postzygotic de novo gene conversion event that resulted in the deletion of the paternally-inherited WT copy of CYP21A2 and led to somatic mosaicism for two cell lines in both twins. Due to this de novo event, the zygote carried two cell lines before twinning (Fig. 3). Cell line 1 would result in an individual being a carrier of CAH. Cell line 2 is typically associated with a salt-wasting $\mathrm{CAH}$ phenotype.

After twinning, both cell lines appear to have been passed to both twins, but resulted in discordant phenotypes. Twin A presented with a biochemical phenotype consistent with salt-wasting $\mathrm{CAH}$, but she did not have physical manifestations requiring surgery. On the other hand, Twin B, presented with, and has maintained, a normal biochemical phenotype, but was found to have a distal urogenital sinus.

These discordant biochemical phenotypes are likely due to the frequency and distribution of the mutant 
cell line 2 in the twins' cells and tissues. Although the authors can only hypothesize, Twin A may have more cells derived from the de novo cell line, or Twin A's adrenal glands may contain more of cell line 2, while Twin B's may contain more of cell line 1 . Only whole blood samples were available for testing; however, it has been suggested that, because $95 \%$ of monochorionic twins exchange blood in utero through vascular anastomoses in their shared placenta, blood mosaicism may result in monochorionic twins $(8,10,11)$. Therefore, future testing of fibroblast cells, or another non-blood-derived sample type, may help to rule out blood-confined mosaicism in Twin B. However, even with testing of additional sample types, the state of mosaicism in the twins' adrenal glands cannot be assessed to test these hypotheses. In addition, one cannot rule out that other genetic factors, such as methylation or additional somatic changes in other genes, may have also played a role in the observed biochemical phenotypic differences observed between Twin A and B.

The cause of the virilization observed in Twin $B$ remains unsolved. However, although she had no biochemical evidence of 21-hydroxylase deficiency, Twin B and Twin A shared a placenta in utero, and thus almost certainly shared circulation $(10,11)$. Due to this shared circulation, there is also a shared hormonal environment $(10,12)$. Twin A would have secreted elevated androgen levels in utero, exposing both twins, and through arteriovenous anastomoses in the placenta, Twin B may have been exposed to more androgens than Twin A (10). Further, even in siblings that share the same genotype, the level of virilization is not always equivalent. Tze et al. reported on a set of dizygotic female infants with 21-hydroxylase CAH where one presented with ambiguous genitalia and the other was thought to have normal genitalia until closer examination revealed an enlargement of the clitoris with no other anomalies. Unfortunately, the twins in Tze et al. were not genotyped, so the affected status of both twins was not confirmed genotypically; however, biochemical results suggested that both dizygotic twins were affected (13).

The results highlighted in this report have important implications for the future management of both Twin A and Twin B, as well as risk counseling for future offspring. Since germline mosaicism in the twins' parents is highly unlikely in this case, the recurrence risk in future pregnancies of the parents is low (approximately 1\%). Twin A and Twin B should continue to be managed appropriately based on their phenotype; however, because both cell lines appear to be present in both twins, they are at risk of having affected children. Genetic counseling should include a discussion that both twins' germline may be mosaic and contain all three CYP21A2 genotypes (WT, c.293-13C >G, or the CYP21A2 deletion). The risk for the twins to pass on a CYP21A2 mutation might range anywhere from $50 \%$ (if their germline is composed only of cell line 1) to $100 \%$ (if their germline is composed only of cell line 2).

In summary, we feel the evidence is compelling that mosaicism resulted in, not only the discordant phenotypes between Twin A and Twin B, but also in the discordance between genotype and biochemical phenotype observed. Intrauterine transfer of sex steroids between the twins is the likely cause for virilization in both twins (with no evidence of glucocorticoid or mineralcorticoid deficiency in Twin B). This result is important for both the continued management of Twin A and Twin B, and for genetic counseling of this family.

This report also illustrates the importance of considering somatic mosaicism as a mechanism for disease in 21-hydroxylase deficient CAH. Indeed, it is interesting to speculate if some of the previously reported genotypephenotype discordance in a minority of patients with the c.293-13C>G/full gene deletion genotype could represent somatic mosaicism $(2,5)$. To our knowledge, this is the first report of suspected somatic mosaicism in $\mathrm{CAH}$, possibly due to the relatively low incidence $(1-2 \%)$ of $d e$ novo genetic events in CYP21A2 cases $(1,3,4,5,6)$.

\section{Supplementary materials}

This is linked to the online version of the paper at https://doi.org/10.1530/ EJE-19-0249.

\section{Declaration of interest}

The authors declare that there is no conflict of interest that could be perceived as prejudicing the impartiality of this case report.

\section{Funding}

This research did not receive any specific grant from any funding agency in the public, commercial or not-for-profit sector.

\section{Informed consent}

Written informed consent for publication of clinical details was obtained from the parent or guardian of the patients.

\section{Author contribution statement}

K F and E G clinically characterized and counseled the patients. H L S performed PCR, Sanger sequencing, and MLPA. D M performed droplet digital PCR. M L K, S K G, and L V H conducted analysis of the results. KW $C$ conducted an additional phasing experiment. M L K prepared the draft manuscript. All authors contributed to the discussion of results, and edited and approved the final manuscript. 


\section{Acknowledgement}

The authors are grateful to and thank the patients and their parents.

\section{References}

1 Wedell A. Molecular genetics of congenital adrenal hyperplasia (21-hydroxylase deficiency): implications for diagnosis, prognosis and treatment. Acta Paediatrica 199887 159-164. (https://doi. org/10.1080/08035259850157598)

2 New MI, Abraham M, Gonzalez B, Dumic M, Razzaghy-Azar M, Chitayat D, Sun L, Zaidi M, Wilson RC \& Yuen T. Genotypephenotype correlation in 1507 families with congenital adrenal hyperplasia owing to 21-hydroxylase deficiency. PNAS 2013110 2611-2616. (https://doi.org/10.1073/pnas.1300057110)

3 Krone N, Braun A, Roscher AA, Knorr D \& Schwarz HP. Predicting phenotype in steroid 21-hydroxylase deficiency? Comprehensive genotyping in 155 unrelated, well defined patients from Southern Germany. Journal of Clinical Endocrinology and Metabolism 200085 1059-1065. (https://doi.org/10.1210/jcem.85.3.6441)

4 Stikkelbroeck NMML, Hoefsloot LH, De Wijs IJ, Otten BJ, Hermus ARMM \& Sistermans EA. CYP21 gene mutation analysis in 198 patients with 21-hydroxylase deficiency in the Netherlands: six novel mutations and a specific cluster of four mutations. Journal of Clinical Endocrinology and Metabolism 200388 3852-3859. (https:// doi.org/10.1210/jc.2002-021681)

5 Finkielstain GP, Chen W, Mehta SP, Fujimura FK, Hanna RM, Van Ryzin C, McDonnel NB \& Merke DP. Comprehensive genetic analysis of 182 unrelated families with congenital adrenal hyperplasia due to 21-hydroxylase deficiency. Journal of Clinical Endocrinology and Metabolism 201196 E161-E172. (https://doi. org/10.1210/jc.2010-0319)
6 Marino R, Ramirez P, Galeano J, Perez Garrido N, Rocco C, Ciaccio M, Warman DM, Guercio G, Chaler E, Maceiras M et al. Steroid 21-hydroxylase gene mutational spectrum in 454 Argentinean patients: genotype-phenotype correlation in a large cohort of patients with congenital adrenal hyperplasia. Clinical Endocrinology 201175 427-435. (https://doi.org/10.1111/j.1365-2265.2011.04123.x)

7 de Carvalho DF, Miranda MC, Gomes LG, Madureira G, Marcondes JAM, Billerbeck AEC, Rodrigues AS, Presti PF, Kuperman H, Damiani D et al. Molecular CYP21A2 diagnosis in 480 Brazilian patients with congenital adrenal hyperplasia before newborn screening introduction. European Journal of Endocrinology 2016175 107-116. (https://doi.org/10.1530/EJE-16-0171)

8 Kaplan L, Foster R, Shen Y, Parry DM, McMaster ML, O'Leary MC $\&$ Gusella JF. Monozygotic twins discordant for neurofibromatosis 1. American Journal of Medical Genetics: Part A 2010 152A 601-606. (https://doi.org/10.1002/ajmg.a.33271)

9 Vogt J, Kohlhase J, Morlot S, Kluwe L, Mautner VF, Cooper DN \& Kehrer-Sawatzki H. Monozygotic twins discordant for neurofibromatosis Type 1 due to a postzygotic NF1 gene mutation. Human Mutation 2011 32 E2134-E2147. (https://doi.org/10.1002/humu.21476)

10 Lewi L, Deprest J \& Hecher K. The vascular anastomoses in monochorionic twin pregnancies and their clinical consequences. American Journal of Obstetrics and Gynecology 2013208 19-30. (https://doi.org/10.1016/j.ajog.2012.09.025)

11 Denbow ML \& Fisk NM. The consequences of monochorionic placentation. Baillière's Clinical Obstetrics and Gynaecology 199812 37-51. (https://doi.org/10.1016/s0950-3552(98)80038-2)

12 Mains LM, Lathi RB, Burney RO \& Dahan MH. Serum total testosterone levels in a patient with late onset 21-hydroxylase deficiency and a twin gestation. Fertility and Sterility $2007 \mathbf{8 7} 1212$. e5-1212.e8. (https://doi.org/10.1016/j.fertnstert.2006.07.1545)

13 Tze WJ, Fisher JN \& Crigler Jr JF. Congenital adrenal hyperplasia: case report in dizygotic twins. Pediatrics 197250 137-139.

Received 9 April 2019

Revised version received 11 November 2019

Accepted 3 December 2019 